Article

\title{
Comparison of Clinical Manifestations,
} Antimicrobial Susceptibility Patterns, and Mutations of Fluoroquinolone Target Genes between Elizabethkingia meningoseptica and Elizabethkingia
anophelis Isolated in Taiwan

\author{
Jiun-Nong Lin ${ }^{1,2,3, * \mathbb{D}}$, Chung-Hsu Lai ${ }^{1,2}$, Chih-Hui Yang ${ }^{4}$ and Yi-Han Huang ${ }^{1}$ \\ 1 School of Medicine, College of Medicine, I-Shou University, Kaohsiung 824, Taiwan; \\ laich6363@yahoo.com.tw (C.-H.L.); je091410show@hotmail.com (Y.-H.H.) \\ 2 Division of Infectious Diseases, Department of Internal Medicine, E-Da Hospital, I-Shou University, \\ Kaohsiung 824, Taiwan \\ 3 Department of Critical Care Medicine, E-Da Hospital, I-Shou University, Kaohsiung 824, Taiwan \\ 4 Department of Biological Science and Technology, Meiho University, Pingtung 912, Taiwan; \\ puppylovefu@gmail.com \\ * Correspondence: jinoli@kmu.edu.tw; Tel: +886-7-615-0011-251469; Fax: +886-7-615-0928
}

Received: 19 November 2018; Accepted: 10 December 2018; Published: 11 December 2018

\begin{abstract}
Elizabethkingia meningoseptica and Elizabethkingia anophelis are two major pathogens in the genus Elizabethkingia. Studies have revealed that Elizabethkingia anophelis is frequently misidentified as E. meningoseptica. Therefore, our aim was to explore the clinical and molecular differences between these two species. The database of a clinical microbiology laboratory in a university-affiliated hospital of Taiwan was searched to identify patients with Elizabethkingia infections between January 2005 and June 2018. Species were reidentified using 16S ribosomal RNA gene sequencing. Twenty E. meningoseptica and 72 E. anophelis samples were collected from consecutive patients. E. meningoseptica was significantly more frequently isolated from the cerebrospinal fluid than was E. anophelis. The most susceptible antibiotic for all Elizabethkingia isolates was minocycline (91.3\%), followed by levofloxacin $(52.2 \%)$, tigecycline $(23.9 \%)$, and piperacillin tazobactam $(23.9 \%)$. Compared with E. anophelis, E. meningoseptica was significantly less susceptible to piperacillin tazobactam, minocycline, and levofloxacin. Regarding nonsynonymous substitutions in the quinolone-resistance determining regions of DNA gyrase, six sites were recognized in E. meningoseptica and one site was recognized in E. anophelis. E. meningoseptica had a significantly higher rate of fluoroquinolone target gene mutations than did E. anophelis. Because of less susceptibility to multiple antibiotics than E. anophelis, empirical antimicrobial therapy of E. meningoseptica should be more rigorous.
\end{abstract}

Keywords: Elizabethkingia meningoseptica; Elizabethkingia anophelis; fluoroquinolone; quinoloneresistance determining region; drug resistance

\section{Introduction}

Elizabethkingia, which is frequently distributed in the natural environments of soil and water, is a genus of aerobic, gram-negative, non-spore-forming, nonmotile, and nonfermenting bacilli $[1,2]$. This genus has been sporadically reported to cause severe infections in humans, particularly in neonates and immunocompromised patients [3-9]. Presently, six species are included in the genus Elizabethkingia, namely E. meningoseptica, E. miricola, E. anophelis, E. bruuniana, E. ursingii, and E. occulta [3]. Among these species, E. meningoseptica is the most well known [4]. However, E. anophelis 
has been increasingly reported to cause outbreaks in Singapore [5], Hong Kong [6], Taiwan [7], and the United States [8,9]. Other species are rarely reported to cause human infections.

Studies have demonstrated that some species of Elizabethkingia could not be accurately identified using traditional biochemical-based phenotyping methods or matrix-assisted laser desorption ionization-time of flight mass spectrometry (MALDI-TOF MS) platforms with a standard reference spectrum database $[6,10]$. In these microbial identification platforms, E. meningoseptica could be correctly identified. Nevertheless, E. anophelis is not included in the identification database, and this species is usually misidentified as E. meningoseptica by these machines $[6,10]$. The accurate identification of E. anophelis species relies on 16S ribosomal RNA (rRNA) gene sequencing or MALDI-TOF platforms with a "Research Use Only" database [6,8,10,11]. However, most studies investigating Elizabethkingia have used unreliable microbial identification methods; consequently, these studies actually presented the clinical or molecular characteristics of all Elizabethkingia species rather than those of each species.

In this study, to precisely explore the genuine epidemiology, clinical characteristics, and antimicrobial susceptibility patterns of E. meningoseptica and E. anophelis, we used 16S rRNA gene sequencing to accurately identify the species of Elizabethkingia. Subsequently, we compared E. meningoseptica and E. anophelis. Because fluoroquinolones were suggested to be an empirical antimicrobial therapy for Elizabethkingia infections [6], we also examined gene alteration in the quinolone-resistance determining regions (QRDRs) of E. meningoseptica and E. anophelis and investigated the association between QRDR mutations and fluoroquinolone resistance.

\section{Materials and Methods}

\subsection{Study Setting and Design}

This study was approved by the Institutional Review Board of E-Da Hospital (EMRP-106-105) and was conducted in accordance with the Declaration of Helsinki and national and institutional standards. The computer database of the clinical microbial laboratory in a 1000-bed university-affiliated medical center in Taiwan was searched for cultures that yielded Elizabethkingia species between January 2005 and June 2018. The API/ID32 Phenotyping Kits (bioMérieux, Marcy l'Etoile, France) and VITEK MS System (bioMérieux, Marcy l'Etoile, France) were used for microbial identification by the clinical microbial laboratory during 2005-2013 and 2014-2018, respectively. All isolates were stored as glycerol stocks at $-80{ }^{\circ} \mathrm{C}$ until use. The species of Elizabethkingia was reidentified using $16 \mathrm{~S}$ rRNA gene sequencing. Patients infected with E. meningoseptica and E. anophelis were included in this study. Clinical characteristics and outcomes were collected from the medical records. Inappropriate empirical antimicrobial therapy was defined as nonsusceptibility of the isolates to the empirically prescribed antibiotics. Shock was defined as systolic pressure of $<90 \mathrm{~mm} \mathrm{Hg}$, a reduction of $40 \mathrm{~mm} \mathrm{Hg}$ in systolic blood pressure from baseline, or a condition requiring inotropic agents to maintain blood pressure.

\subsection{Species Identification Using $16 S$ rRNA Gene Sequencing}

After thawing, the frozen bacteria were subcultured on tryptic soy agar with $5 \%$ sheep blood (Becton Dickinson, Sparks, MD, USA). The colony was then inoculated on fresh tryptic soy agar with $5 \%$ sheep blood for overnight culture. The total DNA of fresh colonies was prepared using a Wizard Genomic DNA Purification Kit (Promega, Madison, WI, USA). The primers and protocols for amplification and sequencing of the $16 \mathrm{~S}$ rRNA gene are listed in Supplementary Table S1 [7,10,12,13]. The assembled sequences of $16 \mathrm{~S}$ rRNA were submitted to the National Center for Biotechnology Information website for comparison with their nucleotide sequences in GenBank sequence databases using the Basic Local Alignment Search Tool (https:/ /blast.ncbi.nlm.nih.gov/Blast.cgi). The similarity of $16 \mathrm{~S}$ rRNA sequences of isolates to the type strains in the GenBank sequence databases was examined (Supplementary Table S2). The species of Elizabethkingia were determined if the isolates shared $\geq 99.5 \%$ of 16S rRNA sequence identity with the type strains in GenBank [14]. The sequences of 16S rRNA were aligned using Clustal W with default options in MEGA7 [15]. Genetic relationships were inferred using 
the Maximum Likelihood method based on the JC69 model [15]. Phylogenetic tree was constructed in Dendroscope [16].

\subsection{Antimicrobial Susceptibility Testing}

The minimum inhibitory concentrations (MICs) of 18 antibiotics were determined using the broth microdilution method with Sensititre 96-well panels as per the manufacturer's instructions (Thermo Fisher Scientific/Trek Diagnostics Systems, Oakwood Village, OH, USA). The susceptibilities were interpreted according to the criteria of "other non-Enterobacteriaceae" in the Clinical \& Laboratory Standards Institute guidelines [17]. The MIC of tigecycline was interpreted according to the Enterobacteriaceae susceptibility breakpoints of the US Food and Drug Administration (susceptible MIC, $\leq 2 \mathrm{mg} / \mathrm{L}$; intermediate MIC, $4 \mathrm{mg} / \mathrm{L}$; resistant MIC, $\geq 8 \mathrm{mg} / \mathrm{L}$ ) [18].

\subsection{Identification of Mutations in the QRDRs}

The primers and amplification conditions for QRDRs in gyrA, gyrB, parC, and parE of E. meningoseptica and E. anophelis are listed in Supplementary Table S1. The names and GenBank accession numbers of strains for comparison of QRDRs are listed in Supplementary Table S2.

\subsection{Data Analysis}

The data were analyzed using SPSS version 24.0 (IBM, Armonk, NY, USA). Categorical variables were calculated using the chi-squared test or Fisher exact test as appropriate. Variables associated with mortality were examined using univariate analyses. All variables were included in a logistic regression model of multivariate analysis with backward stepwise methods using the likelihood ratio. The significance of variables was calculated by odds ratios (ORs), $95 \%$ confidence intervals (CIs), and a two-tailed $p$-value. A $p$-value of $<0.05$ was considered statistically significant.

\section{Results}

\subsection{Species Identification}

During the investigation period, 103 nonduplicated Elizabethkingia isolates were collected by the clinical microbiology laboratory. Three isolates died after thawing. After comparisons with the $16 \mathrm{~S}$ rRNA gene sequences of type strains in GenBank (Supplementary Table S2), 20 isolates were identified as E. meningoseptica, 72 as E. anophelis, and eight as E. bruuniana. The $16 \mathrm{~S}$ rRNA-based phylogenetic analysis clearly discriminated the species of these isolates (Figure 1). 


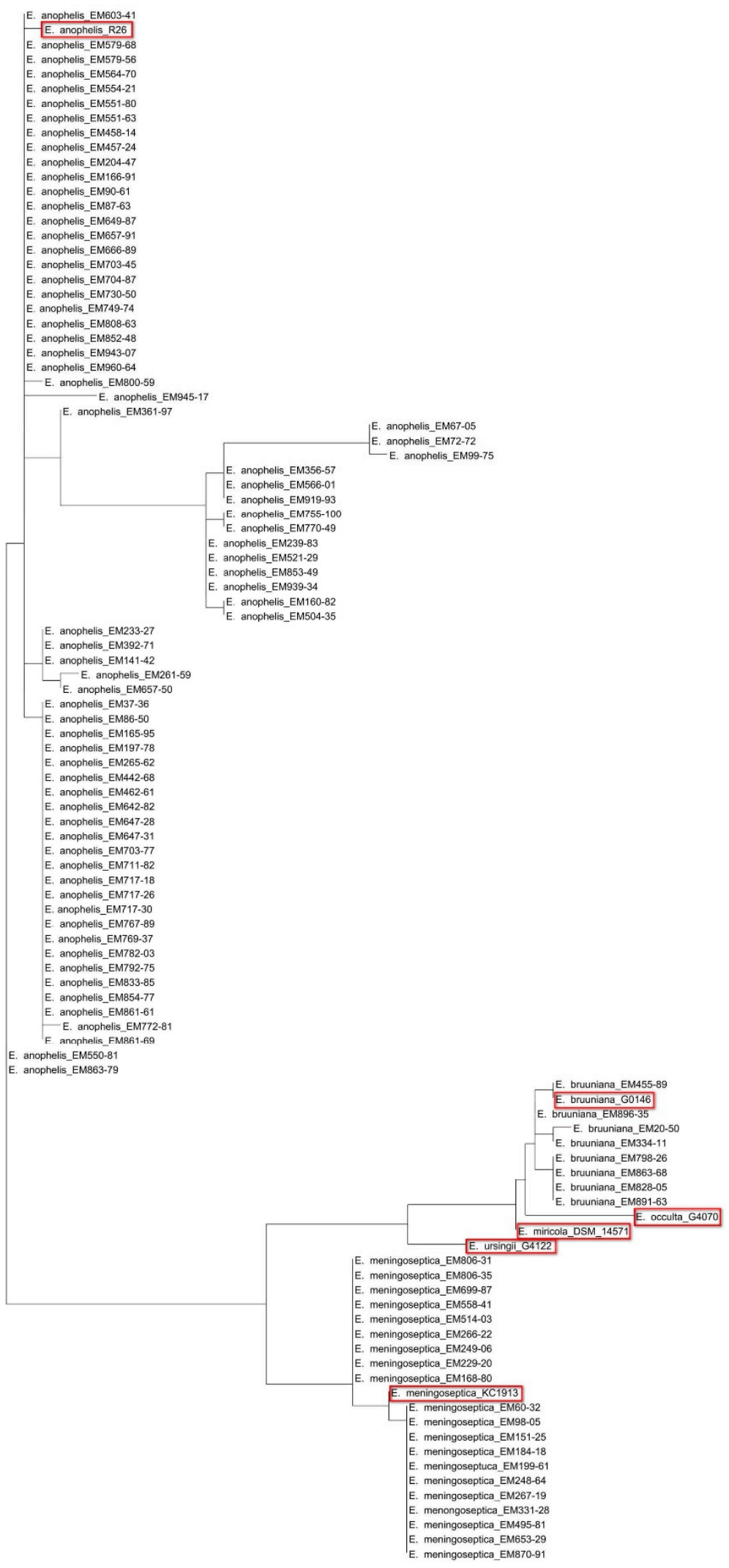

Figure 1. The phylogenetic tree based on 16S rRNA gene sequences among 20 Elizabethkingia meningoseptica isolates, 72 Elizabethkingia anophelis isolates, eight Elizabethkingia bruuniana isolates, and the six type strains of the Elizabethkingia genus. The tree is drawn to scale, with branch lengths in the same units as those of the evolutionary distances used to infer the phylogenetic tree. Red rectangles: the six type strains of the Elizabethkingia genus. 


\subsection{Site of Isolation}

Of these E. meningoseptica and E. anophelis isolates, the most common site of isolation was blood $(60.9 \%)$, followed by the respiratory tract $(12 \%)$, the tip of the central venous catheter $(8.7 \%)$, bile $(6.5 \%)$, and urine (4.3\%) (Table 1). The other sites of isolation included cerebrospinal fluid $(2.2 \%)$, ascites $(2.2 \%)$, wounds/abscesses $(2.2 \%)$, and pleural effusion (1.1\%). Compared with E. anophelis, E. meningoseptica was significantly more frequently isolated from the cerebrospinal fluid $(p=0.045)$.

Table 1. The isolation sites of E. meningoseptica and E. anophelis.

\begin{tabular}{cccccc}
\hline Site of Isolation & All (n= 92) & $\begin{array}{c}\text { Number of Episodes (\%) } \\
\text { E. meningoseptica } \\
(\boldsymbol{n}=\mathbf{2 0})\end{array}$ & $\begin{array}{c}\text { E. anophelis } \\
(\boldsymbol{n}=\mathbf{7 2})\end{array}$ & OR (95\% CI) & p-Value \\
\hline Cerebrospinal fluid & $2(2.2)$ & $2(10)$ & 0 & & 0.045 \\
Pleural effusion & $1(1.1)$ & 0 & $1(1.4)$ & & 0.999 \\
Respiratory tract & $11(12)$ & $1(5)$ & $10(13.9)$ & $0.33(0.04-2.72)$ & 0.445 \\
Ascites & $2(2.2)$ & 0 & $2(2.8)$ & & 0.999 \\
Bile & $6(6.5)$ & $1(5)$ & $5(6.9)$ & $0.71(0.08-6.41)$ & 0.999 \\
Blood & $56(60.9)$ & $14(70)$ & $42(58.3)$ & $1.67(0.58-4.84)$ & 0.441 \\
Urine & $8(8.7)$ & $1(5)$ & $7(9.7)$ & $0.49(0.06-4.22)$ & 0.681 \\
Tip of central venous catheter & $4(4.3)$ & $1(5)$ & $3(4.2)$ & $1.21(0.12-12.3)$ & 0.999 \\
Wound/Abscess & $2(2.2)$ & 0 & $2(2.8)$ & & 0.999 \\
\hline
\end{tabular}

Abbreviations: OR, odds ratio; $\mathrm{CI}$, confidence interval.

\subsection{Clinical Characteristics of Elizabethkingia Infections}

These 92 nonrepeated Elizabethkingia samples were isolated from 92 consecutive patients. Of these patients, men accounted for $69.6 \%$ and the median age was 61 years (Table 2). Chronic illness was found in $78.3 \%$ of the patients. The most common comorbidity was malignancy (43.5\%), followed by hypertension (28.3\%) and diabetes mellitus (26.1\%). The majority of infections $(89.1 \%)$ were attributed to healthcare-associated infection. Shock was recognized in $45.7 \%$ of the patients; and $47.8 \%$ of the patients were admitted to intensive care units. Empirical antibiotics included $\beta$-lactams $(44.6 \%)$, $\beta$-lactam/lactamase inhibitors $(21.7 \%)$, ciprofloxacin $(10.9 \%)$, levofloxacin $(26.1 \%)$, carbapenems $(18.5 \%)$, aminoglycosides $(9.8 \%)$, tigecycline $(8.7 \%)$, and colistin $(6.5 \%)$, either individually or in combination. Among these empirical antibiotic treatments, $80.4 \%$ were considered inappropriate empirical antimicrobial therapies. The overall case fatality rate of Elizabethkingia infections was $27.2 \%$.

When patients with E. meningoseptica and E. anophelis infections were compared, no statistical differences were observed in the onset of age, sex, comorbidity, laboratory data, or case-fatality rate. Levofloxacin was more commonly used in patients with E. anophelis infection $(p=0.015)$. Inappropriate empirical antimicrobial therapy was more frequently recognized in patients with E. meningoseptica infections than in those with E. anophelis infections $(p=0.01$; Table 2).

Table 2. Demographic characteristics, clinical information, and outcome of patients with E. meningoseptica and E. anophelis infections.

\begin{tabular}{|c|c|c|c|c|c|}
\hline \multirow[b]{2}{*}{ Characteristics } & \multirow[b]{2}{*}{ All $(n=92)$} & \multicolumn{2}{|c|}{ Number of Episodes (\%) } & \multirow[b]{2}{*}{ OR $(95 \% \mathrm{CI})$} & \multirow[b]{2}{*}{$p$-Value } \\
\hline & & $\begin{array}{c}\text { E. meningoseptica } \\
(n=20)\end{array}$ & $\begin{array}{l}\text { E. anophelis } \\
(n=72)\end{array}$ & & \\
\hline \multicolumn{6}{|l|}{ Sex } \\
\hline Male & $64(69.6)$ & $15(75)$ & $49(68.1)$ & $1.41(0.46-4.35)$ & 0.55 \\
\hline Female & $28(30.4)$ & $5(25)$ & $23(31.9)$ & $0.71(0.23-2.19)$ & 0.55 \\
\hline \multicolumn{6}{|l|}{ Age } \\
\hline Range (year) & $3-89$ & $18-80$ & $3-89$ & & \\
\hline Median (year) & 61 & 61 & 62.5 & & \\
\hline Mean \pm standard deviation (year) & $61.1 \pm 17$ & $56.6 \pm 15.6$ & $62.4 \pm 17.3$ & & 0.179 \\
\hline
\end{tabular}


Table 2. Cont.

\begin{tabular}{|c|c|c|c|c|c|}
\hline \multirow[b]{2}{*}{ Characteristics } & \multirow[b]{2}{*}{ All $(n=92)$} & \multicolumn{2}{|c|}{ Number of Episodes (\%) } & \multirow[b]{2}{*}{ OR $(95 \% \mathrm{CI})$} & \multirow[b]{2}{*}{$p$-Value } \\
\hline & & $\begin{array}{l}\text { E. meningoseptica } \\
\quad(n=20)\end{array}$ & $\begin{array}{l}\text { E. anophelis } \\
(n=72)\end{array}$ & & \\
\hline \multicolumn{6}{|l|}{ Comorbidity } \\
\hline Diabetes mellitus & $24(26.1)$ & $6(30)$ & $18(25)$ & $1.29(0.43-3.84)$ & 0.652 \\
\hline Hypertension & $26(28.3)$ & $4(20)$ & $22(30.6)$ & $0.57(0.17-1.9)$ & 0.354 \\
\hline End-stage renal disease & $5(5.4)$ & $1(5)$ & $4(5.6)$ & $0.9(0.09-8.49)$ & 0.999 \\
\hline Malignancy & $40(43.5)$ & $8(40)$ & $32(44.4)$ & $0.83(0.3-2.28)$ & 0.723 \\
\hline Liver cirrhosis & $8(8.7)$ & $3(15)$ & $5(6.9)$ & $2.37(0.51-10.89)$ & 0.365 \\
\hline Chronic obstructive pulmonary disease & $9(9.8)$ & 0 & $9(12.5)$ & & 0.197 \\
\hline \multicolumn{6}{|l|}{ Type of infection acquisition } \\
\hline Community-acquired infection & $9(9.8)$ & 0 & $9(12.5)$ & & 0.197 \\
\hline Healthcare-associated infection & $83(90.2)$ & 20 & $63(87.5)$ & & 0.197 \\
\hline \multicolumn{6}{|l|}{ Laboratory data } \\
\hline White blood cell count (cells $/ \mathrm{mm}^{3}$ ) & $13,281 \pm 8740$ & $13,353 \pm 6687$ & $13,261 \pm 9271$ & & 0.967 \\
\hline Hemoglobin $(\mathrm{g} / \mathrm{dL})$ & $10.1 \pm 2.1$ & $9.8 \pm 2.4$ & $10.1 \pm 2.1$ & & 0.585 \\
\hline Platelet count $\left(\times 1000\right.$ cells $\left./ \mathrm{mm}^{3}\right)$ & $228,570 \pm 131,056$ & $216,550 \pm 157,332$ & $231,900 \pm 123,846$ & & 0.69 \\
\hline Serum creatinine $(\mathrm{mg} / \mathrm{dL})$ & $1.8 \pm 1.7$ & $1.6 \pm 1.3$ & $1.9 \pm 1.8$ & & 0.584 \\
\hline \multicolumn{6}{|l|}{ Empirical antimicrobial therapy } \\
\hline$\beta$-lactams & $41(44.6)$ & $11(55)$ & $30(41.7)$ & $1.71(0.63-4.64)$ & 0.289 \\
\hline$\beta$-lactam/lactamase inhibitors & $20(21.7)$ & $4(20)$ & $16(22.2)$ & $0.88(0.26-2.99)$ & 0.999 \\
\hline Ciprofloxacin & $10(10.9)$ & $1(5)$ & $9(12.5)$ & $0.37(0.04-3.1)$ & 0.685 \\
\hline Levofloxacin & $24(26.1)$ & $1(5)$ & $23(31.9)$ & $0.11(0.01-0.89)$ & 0.015 \\
\hline Carbapenems & 17 (18.5) & $4(20)$ & $13(18.1)$ & $1.14(0.33-3.96)$ & 0.999 \\
\hline Aminoglycosides & $9(9.8)$ & $3(15)$ & $6(8.3)$ & $1.94(0.44-8.57)$ & 0.402 \\
\hline Tigecycline & $8(8.7)$ & $2(10)$ & $6(8.3)$ & $1.22(0.23-6.58)$ & 0.999 \\
\hline Colistin & $6(6.5)$ & $1(5)$ & $5(6.9)$ & $0.71(0.08-6.41)$ & 0.999 \\
\hline Inappropriate empirical antimicrobial therapy & $74(80.4)$ & $20(100)$ & $54(75)$ & & 0.01 \\
\hline Shock & $42(45.7)$ & $9(45)$ & $33(45.8)$ & $0.97(0.36-2.62)$ & 0.999 \\
\hline Admission to intensive care unit & $44(47.8)$ & $9(45)$ & $35(48.6)$ & $0.87(0.32-2.34)$ & 0.775 \\
\hline Case fatality & $25(27.2)$ & $6(30)$ & $19(26.4)$ & $1.2(0.4-3.56)$ & 0.748 \\
\hline
\end{tabular}

Abbreviations: OR, odds ratio; $\mathrm{CI}$, confidence interval.

\subsection{Factors Associated with Mortality}

Univariate analysis showed that patients with liver cirrhosis $(p=0.032)$ and inappropriate empirical antimicrobial therapy $(p=0.02)$ had a higher mortality rate. Multivariate logistic regression analysis revealed that inappropriate empirical antimicrobial therapy was an independent risk factor for mortality in patients with Elizabethkingia infections (adjusted OR, 12.45; 95\% CI, 1.33-116.77; $p=0.027$; Table 3).

Table 3. Factors associated with mortality in patients with Elizabethkingia infections.

\begin{tabular}{|c|c|c|c|c|c|c|}
\hline \multirow{2}{*}{ Factor } & \multicolumn{2}{|c|}{ Outcome } & \multicolumn{2}{|c|}{ Univariate Analysis } & \multicolumn{2}{|c|}{ Multivariate Analysis } \\
\hline & Died & Survived & OR $(95 \%$ CI $)$ & $p$-Value & OR $(95 \%$ CI) & $p$-Value \\
\hline \multicolumn{7}{|l|}{ All Isolates $(n=92)$} \\
\hline \multicolumn{7}{|l|}{ Species } \\
\hline E. meningoseptica & $6(24)$ & $14(20.9)$ & $1.2(0.4-3.56)$ & 0.748 & $1.76(0.5-6.16)$ & 0.38 \\
\hline E. anophelis & $19(76)$ & 53 (79.1) & & 0.748 & $0.57(0.16-2)$ & 0.38 \\
\hline Age $\geq 65$ years & $12(48)$ & $29(43.3)$ & $1.21(0.48-3.04)$ & 0.686 & $2.89(0.86-9.74)$ & 0.087 \\
\hline Diabetes mellitus & $8(32)$ & $16(23.9)$ & $1.5(0.55-4.12)$ & 0.43 & $1.56(0.44-5.49)$ & 0.491 \\
\hline Hypertension & $4(16)$ & $22(32.8)$ & $0.39(0.12-1.27)$ & 0.111 & $0.27(0.07-1.11)$ & 0.069 \\
\hline End-stage renal disease & $1(4)$ & $4(6)$ & $0.66(0.07-6.17)$ & 0.999 & $0.45(0.03-6.77)$ & 0.566 \\
\hline Malignancy & $12(48)$ & $28(41.8)$ & $1.29(0.51-3.24)$ & 0.593 & $1.11(0.35-3.47)$ & 0.86 \\
\hline Liver cirrhosis & $5(20)$ & $3(4.5)$ & $5.33(1.17-24.31)$ & 0.032 & $4.67(0.9-24.3)$ & 0.067 \\
\hline Chronic obstructive pulmonary disease & $4(16)$ & $5(7.5)$ & $2.36(0.58-9.62)$ & 0.248 & $1.98(0.28-18.87)$ & 0.492 \\
\hline
\end{tabular}


Table 3. Cont.

\begin{tabular}{|c|c|c|c|c|c|c|}
\hline \multirow{2}{*}{ Factor } & \multicolumn{2}{|c|}{ Outcome } & \multicolumn{2}{|c|}{ Univariate Analysis } & \multicolumn{2}{|c|}{ Multivariate Analysis } \\
\hline & Died & Survived & OR $(95 \% \mathrm{CI})$ & $p$-Value & OR $(95 \% \mathrm{CI})$ & $p$-Value \\
\hline \multicolumn{7}{|l|}{ E. meningoseptica $(n=20)$} \\
\hline Sex, male & $5(83.3)$ & $10(71.4)$ & $2(0.17-22.95)$ & 0.999 & & 0.999 \\
\hline \multicolumn{7}{|l|}{ Underlying disease } \\
\hline Diabetes mellitus & $2(33.3)$ & $4(28.6)$ & $1.25(0.16-9.77)$ & 0.999 & $4.5(0.31-65.23)$ & 0.27 \\
\hline Malignancy & $2(33.3)$ & $6(42.9)$ & $0.67(0.09-4.93)$ & 0.999 & $0.68(0.03-17.96)$ & 0.814 \\
\hline Liver cirrhosis & $2(33.3)$ & $1(7.1)$ & $6.5(0.46-91.92)$ & 0.202 & $5.11(0.3-87.96)$ & 0.261 \\
\hline \multirow{2}{*}{$\begin{array}{c}\text { Chronic obstructive pulmonary disease } \\
\text { Inappropriate empirical antimicrobial } \\
\text { therapy }\end{array}$} & 0 & 0 & & & & \\
\hline & $6(100)$ & 0 & & & & \\
\hline \multicolumn{7}{|l|}{ E. anophelis $(n=72)$} \\
\hline \multicolumn{7}{|l|}{ Underlying disease } \\
\hline Hypertension & $4(21.1)$ & $18(34)$ & $0.52(0.15-1.79)$ & 0.295 & $0.36(0.09-1.46)$ & 0.153 \\
\hline End-stage renal disease & $1(5.3)$ & $3(5.7)$ & $0.93(0.09-9.48)$ & 0.999 & $0.66(0.04-10.92)$ & 0.773 \\
\hline Malignancy & $10(52.6)$ & $22(41.5)$ & $1.57(0.55-4.49)$ & 0.403 & $1.3(0.34-4.98)$ & 0.706 \\
\hline Liver cirrhosis & $3(15.8)$ & $2(3.8)$ & $4.78(0.73-31.19)$ & 0.111 & $3.4(0.51-22.5)$ & 0.204 \\
\hline \multirow{2}{*}{$\begin{array}{c}\text { Chronic obstructive pulmonary disease } \\
\text { Inappropriate empirical antimicrobial } \\
\text { therapy }\end{array}$} & $4(21.1)$ & $5(9.4)$ & $2.56(0.61-10.77)$ & 0.231 & $3.06(0.59-15.9)$ & 0.183 \\
\hline & $18(94.7)$ & $36(67.9)$ & $8.5(1.05-69.04)$ & 0.021 & $8.5(1.05-69.04)$ & 0.045 \\
\hline
\end{tabular}

Abbreviations: OR, odds ratio; CI, confidence interval.

\subsection{Antimicrobial Susceptibility Testing}

The MICs and susceptibilities of Elizabethkingia are shown in Table 4. Except for piperacillin and piperacillin tazobactam, high MIC values were detected in $\beta$-lactams, $\beta$-lactam $/ \beta$-lactam inhibitors, carbapenems, aminoglycosides, tetracycline, and trimethoprim-sulfamethoxazole. The Elizabethkingia isolates were most susceptible to minocycline $(91.3 \%)$, followed by levofloxacin $(52.2 \%)$, tigecycline (23.9\%), and piperacillin tazobactam (23.9\%).

When the susceptibility of each species was compared, E. meningoseptica generally had higher MIC values to multiple antibiotics than did E. anophelis. The isolates of E. meningoseptica were most susceptible to minocycline $(60 \%)$, levofloxacin (30\%), piperacillin (15\%), and tigecycline $(15 \%)$. Regarding the isolates of E. anophelis, the most susceptible antibiotic was minocycline (100\%), followed by levofloxacin $(58.3 \%)$, piperacillin tazobactam $(30.6 \%)$, tigecycline $(26.4 \%)$, and piperacillin $(19.4 \%)$. Compared with E. anophelis, E. meningoseptica exhibited significantly lower susceptible rates to piperacillin tazobactam $(p=0.02)$, minocycline $(p<0.001)$, and levofloxacin $(p=0.025)$. Moreover, six E. meningoseptica isolates were resistant to all tested antibiotics, but none E. anophelis was resistant to all antibiotics $(p<0.001$; Table 5). 
Table 4. The minimum inhibitory concentration and susceptibility of Elizabethkingia in this study.

\begin{tabular}{|c|c|c|c|c|c|c|c|c|c|c|c|}
\hline \multirow{2}{*}{ Characteristics } & \multicolumn{3}{|c|}{ All Elizabethkingia $(n=92)$} & \multicolumn{3}{|c|}{ E. meningoseptica $(n=20)$} & \multicolumn{3}{|c|}{ E. anophelis $(n=72)$} & \multirow{2}{*}{ OR $(95 \% \text { CI })^{d}$} & \multirow{2}{*}{$p$-Value ${ }^{\mathrm{d}}$} \\
\hline & $\mathrm{MIC}_{50}{ }^{\mathrm{a}}$ & $\mathrm{MIC}_{90}{ }^{\mathrm{b}}$ & $\mathrm{S}, n(\%)^{\mathrm{c}}$ & $\mathrm{MIC}_{50}{ }^{\mathrm{a}}$ & $\mathrm{MIC}_{90}{ }^{\mathrm{b}}$ & $\mathrm{S}, n(\%)^{\mathrm{c}}$ & $\mathrm{MIC}_{50}{ }^{\mathrm{a}}$ & $\mathrm{MIC}_{90}{ }^{\mathrm{b}}$ & $\mathrm{S}, n(\%)^{\mathrm{c}}$ & & \\
\hline Piperacillin & 64 & $>64$ & 17 (18.5) & 64 & $>64$ & $3(15)$ & 64 & $>64$ & $14(19.4)$ & $0.73(0.19-2.85)$ & 0.756 \\
\hline Piperacillin tazobactam & $128 / 4$ & $>128 / 4$ & $22(23.9)$ & $128 / 4$ & $>128 / 4$ & $1(5)$ & $64 / 4$ & $>128 / 4$ & $22(30.6)$ & $0.12(0.02-0.95)$ & 0.02 \\
\hline Ticarcillin-clavulanic acid & $>64 / 2$ & $>64 / 2$ & 0 & $>64 / 2$ & $>64 / 2$ & 0 & $>64 / 2$ & $>64 / 2$ & 0 & & \\
\hline Ceftazidime & $>16$ & $>16$ & 0 & $>16$ & $>16$ & 0 & $>16$ & $>16$ & 0 & & \\
\hline Cefepime & $>32$ & $>32$ & $1(2.2)$ & $>32$ & $>32$ & 0 & $>32$ & $>32$ & $2(2.8)$ & & 0.999 \\
\hline Ceftriaxone & $>32$ & $>32$ & 0 & $>32$ & $>32$ & 0 & $>32$ & $>32$ & 0 & & \\
\hline Aztreonam & $>16$ & $>16$ & 0 & $>16$ & $>16$ & 0 & $>16$ & $>16$ & 0 & & \\
\hline Imipenem & $>8$ & $>8$ & 0 & $>8$ & $>8$ & 0 & $>8$ & $>8$ & 0 & & \\
\hline Meropenem & $>8$ & $>8$ & 0 & $>8$ & $>8$ & 0 & $>8$ & $>8$ & 0 & & \\
\hline Tobramycin & $>8$ & $>8$ & 0 & $>8$ & $>8$ & 0 & $>8$ & $>8$ & 0 & & \\
\hline Amikacin & $>32$ & $>32$ & $4(4.3)$ & $>32$ & $>32$ & 0 & $>32$ & $>32$ & $4(5.6)$ & & 0.573 \\
\hline Tetracycline & $>8$ & $>8$ & 0 & $>8$ & $>8$ & 0 & $>8$ & $>8$ & 0 & & \\
\hline Minocycline & $<1$ & 4 & 84 (91.3) & 2 & 4 & $12(60)$ & $<1$ & 2 & $72(100)$ & & $<0.001$ \\
\hline Tigecycline & 4 & $>8$ & $22(23.9)$ & 4 & $>8$ & $3(15)$ & 4 & 8 & $19(26.4)$ & $0.49(0.13-1.87)$ & 0.382 \\
\hline Ciprofloxacin & 2 & $>2$ & $9(9.8)$ & $>2$ & $>2$ & $2(10)$ & 2 & $>2$ & $7(9.7)$ & $1.03(0.2-5.4)$ & 0.999 \\
\hline Levofloxacin & 2 & $>8$ & $48(52.2)$ & 8 & $>8$ & $6(30)$ & 2 & $>8$ & $42(58.3)$ & $0.31(0.11-0.89)$ & 0.025 \\
\hline Trimethoprim-sulfamethoxazole & $>4 / 76$ & $>4 / 76$ & $11(12)$ & $>4 / 76$ & $>4 / 76$ & $2(10)$ & $>4 / 76$ & $>4 / 76$ & $9(12.5)$ & $0.78(0.15-3.93)$ & 0.999 \\
\hline
\end{tabular}

Abbreviations: S, susceptible; OR, odds ratio; CI, confidence interval; ${ }^{a} \mathrm{MIC}_{50}$, minimum inhibitory concentration at which $50 \%$ of the isolates tested are inhibited; ${ }^{\mathrm{b}} \mathrm{MIC}_{90}$, minimum inhibitory concentration at which $90 \%$ of the isolates tested are inhibited; ${ }^{\mathrm{c}}$ The number of susceptible isolates and susceptible rates; ${ }^{\mathrm{d}}$ Comparison for the susceptible rates between E. meningoseptica and E. anophelis. 
Table 5. The number of susceptible antibiotics among the 18 tested antibiotics for E. meningoseptica and E. anophelis infections.

\begin{tabular}{cccccc}
\hline $\begin{array}{c}\text { Number of } \\
\text { Susceptible } \\
\text { Antibiotics }\end{array}$ & All Isolates $(\boldsymbol{n = 9 2 )}$ & $\begin{array}{c}\text { Number of Episodes (\%) } \\
\text { n. meningoseptica } \\
(\boldsymbol{n}=\mathbf{2 0})\end{array}$ & $\begin{array}{c}\text { E. anopheles } \\
(\boldsymbol{n}=\mathbf{7 2})\end{array}$ & OR (95\% CI) & p-Value \\
\hline 0 & $6(6.5)$ & $6(30)$ & 0 & & \\
1 & $22(23.9)$ & $5(25)$ & $17(23.6)$ & $0.93(0.29-2.93)$ & 0.009 \\
2 & $28(30.4)$ & $5(25)$ & $23(31.9)$ & $1.41(0.46-4.35)$ & 0.55 \\
3 & $19(20.7)$ & $3(15)$ & $16(22.2)$ & $1.62(0.42-6.23)$ & 0.755 \\
4 & $7(7.6)$ & 0 & $7(9.7)$ & & 0.34 \\
5 & $5(5.4)$ & $1(5)$ & $4(5.6)$ & $1.12(0.12-10.6)$ & 0.999 \\
6 & $3(3.3)$ & 0 & $3(4.2)$ & & 0.999 \\
7 & $2(2.2)$ & 0 & $2(2.8)$ & & 0.999 \\
\hline
\end{tabular}

Abbreviations: OR, odds ratio; $\mathrm{CI}$, confidence interval.

\subsection{Mutations in the QRDRs}

The nonsynonymous substitutions of amino acids in the QRDRs of E. meningoseptica and E. anophelis are shown in Table 6. Isoleucine at position 83 in GyrA $(p<0.001)$, serine at position 95 in $\operatorname{GyrA}(p=0.037)$, arginine at position 452 in $\operatorname{GyrB}(p=0.044)$, and glutamine at position 470 in $\operatorname{GyrB}(p=0.037)$ were associated with levofloxacin nonsusceptibility. No nonsynonymous substitutions were observed in the QRDRs of ParC and ParE. Of the 20 E. meningoseptica isolates, four were found to have amino-acid alteration at position 83 (Ser83Ile) in the QRDR of GyrA. Of the 72 E. anophelis isolates, nine and three possessed Ser83Ile and Ser83Arg in GyrA, respectively. E. meningoseptica had an additional five nonsynonymous alteration sites in the QRDRs, comprising two in GyrA (positions 95 and 102) and three in GyrB (positions 425, 452, and 470). However, E. anophelis had no amino acid alterations at these sites. When the fluoroquinolone target gene mutations were compared, E. meningoseptica had a significantly higher frequency of amino acid alterations at positions 95 and 102 in GyrA and positions 425 and 470 in GyrB than did E. anopheles.

Table 6. Amino acid alternations in the QRDRs of GyrA and GyrB and levofloxacin susceptibility in E. meningoseptica and E. anophelis.

\begin{tabular}{|c|c|c|c|c|c|c|c|}
\hline \multirow[b]{2}{*}{ Amino Acid } & \multicolumn{4}{|c|}{ Susceptibility of Levofloxacin } & \multicolumn{3}{|c|}{ No. of Episode (\%) } \\
\hline & $\begin{array}{l}\text { Susceptible } \\
\quad(n=48)\end{array}$ & $\begin{array}{c}\text { Non-Susceptible } \\
(n=44)\end{array}$ & OR $(95 \% \mathrm{CI})$ & $p$-Value & $\begin{array}{l}\text { E. meningoseptica } \\
\quad(n=20)\end{array}$ & $\begin{array}{c}\text { E. anopheles } \\
(n=72)\end{array}$ & $p$-Value \\
\hline \multicolumn{8}{|c|}{ Position 83 of GyrA } \\
\hline Serine & $48(100)$ & $28(63.6)$ & & $<0.001$ & $16(80)$ & $60(83.3)$ & 0.478 \\
\hline Isoleucine & 0 & $13(29.5)$ & & $<0.001$ & $4(20)$ & $9(12.5)$ & \\
\hline Arginine & 0 & $3(6.8)$ & & 0.105 & 0 & $3(4.2)$ & \\
\hline \multicolumn{8}{|c|}{ Position 95 of GyrA } \\
\hline Serine & $5(10.4)$ & $12(27.3)$ & $0.31(0.1-0.97)$ & 0.037 & $17(85)$ & $72(100)$ & 0.009 \\
\hline Proline & $1(2.1)$ & $2(4.5)$ & $0.45(0.04-5.11)$ & 0.605 & $3(15)$ & 0 & \\
\hline \multicolumn{8}{|c|}{ Position 102 of GyrA } \\
\hline Lysine & $3(6.3)$ & $9(20.5)$ & $0.26(0.07-1.03)$ & 0.055 & $12(60)$ & $72(100)$ & $<0.001$ \\
\hline Glutamine & $3(6.3)$ & $5(11.4)$ & $0.52(0.12-2.32)$ & 0.473 & $8(40)$ & 0 & \\
\hline \multicolumn{8}{|c|}{ Position 425 of GyrB } \\
\hline Isoleucine & $2(4.2)$ & $7(15.9)$ & $0.23(0.05-1.17)$ & 0.081 & $9(45)$ & $72(100)$ & $<0.001$ \\
\hline Lysine & $4(8.3)$ & $7(15.9)$ & $0.48(0.13-1.77)$ & 0.263 & $11(55)$ & 0 & \\
\hline \multicolumn{8}{|c|}{ Position 452 of GyrB } \\
\hline Arginine & $6(12.5)$ & $13(29.5)$ & $0.341(0.12-0.996)$ & 0.044 & $19(95)$ & $72(100)$ & 0.217 \\
\hline Serine & 0 & $1(2.3)$ & & 0.478 & $1(5)$ & 0 & \\
\hline \multicolumn{8}{|c|}{ Position 470 of GyrB } \\
\hline Glutamate & $5(10.4)$ & $12(27.3)$ & $0.31(0.1-0.97)$ & 0.037 & $17(85)$ & $72(100)$ & 0.009 \\
\hline Aspartate & $1(2.1)$ & $2(4.5)$ & $0.45(0.04-5.11)$ & 0.605 & $3(15)$ & 0 & \\
\hline
\end{tabular}

Abbreviations: QRDR, quinolone-resistance determining region; $\mathrm{OR}$, odds ratio; $\mathrm{CI}$, confidence interval.

\section{Discussion}

In this study, we used 16S rRNA gene sequencing as a reference method to identify species of Elizabethkingia collected during the past 13.5 years. We found that E. anophelis, rather than E. meningoseptica, accounted for the majority of human infections in the genus of Elizabethkingia. 
Furthermore, we recognized the differences in antimicrobial susceptibility patterns and amino-acid alterations in the QRDRs between E. meningoseptica and E. anophelis.

Since its first isolation in 1959 by Elizabeth O. King [4], E. meningoseptica has been reported to cause meningitis, pneumonia, bacteremia, and neutropenic fever in humans [2,19-23]. E. anophelis, which was first isolated from the midgut of a mosquito in 2011 [24], has also been reported to cause similar infections to E. meningoseptica [5-9]. Bacteremia has been determined to be a frequent presentation of Elizabethkingia infections [6-9,19-23]. Our study also showed that blood was the most common isolation site of both E. meningoseptica and E. anophelis. Although meningitis caused by $E$. anophelis has been reported [25], our study demonstrated that E. meningoseptica, as its name indicates, was more frequently associated with meningitis than was E. anophelis.

Studies have shown that most patients with Elizabethkingia infections had chronic underlying illnesses, such as diabetes mellitus, cardiovascular disease, chronic renal disease, malignancy, and liver cirrhosis [6-9,19-23]. Our study presents a similar result. The case fatality rate of patients with E. anophelis infection has been approximately $24-34 \%$ in previous reports [6-9]. However, the actual mortality rate of patients with species-confirmed E. meningoseptica is unclear. In our study, the case-fatality rate of E. meningoseptica was $30 \%$, which is similar to that of E. anophelis (26.4\%). We further analyzed the factors affecting mortality. After adjusting the confounding variables in the multivariate analysis model, we noted that inadequate empirical antimicrobial therapy was an independent risk factor for mortality. Therefore, how to choose appropriate empirical antibiotics for patients with Elizabethkingia infections is paramount.

Limited information exists on the antimicrobial susceptibility patterns of Elizabethkingia. As in the notable recent outbreak, several studies have shown that $E$. anophelis was usually resistant to multiple antibiotics, including most $\beta$-lactams, $\beta$-lactam/lactamase inhibitors, carbapenems, and aminoglycoside, but was variably susceptible to piperacillin, piperacillin tazobactam, minocycline, tigecycline, fluoroquinolones, and trimethoprim-sulfamethoxazole $[6-9,26]$. However, no data exist on the antimicrobial susceptibility patterns of E. meningoseptica identified using reliable methods. In our study, all E. anophelis isolates were susceptible to minocycline, and $58.3 \%$ and $30.6 \%$ were susceptible to levofloxacin and piperacillin tazobactam, respectively. By contrast, only $60 \%$ of E. meningoseptica isolates were susceptible to minocycline, $30 \%$ were susceptible to levofloxacin, and $5 \%$ were susceptible to piperacillin tazobactam. E. meningoseptica exhibited a significantly higher nonsusceptible rate to these antibiotics than did E. anophelis. The differences in the antimicrobial susceptibility patterns between E. meningoseptica and E. anophelis have never been presented in the literature. These results suggest that the choice of empirical antimicrobial therapy for E. meningoseptica should differ from that for E. anophelis.

Despite being a recommended antimicrobial therapy for Elizabethkingia infections, our study revealed that numerous Elizabethkingia isolates, particularly E. meningoseptica, were not susceptible to fluoroquinolones. Among the three mechanisms of fluoroquinolone resistance, gene mutations in the QRDRs of $g y r A, g y r B$, parC, and parE are a crucial mechanism of fluoroquinolone resistance [27]. Our study demonstrated that replacement of serine with isoleucine at position 83 (Ser83Ile) in the QRDR of GyrA was recognized both in E. meningoseptica and E. anophelis. Except for this, five additional sites of nonsynonymous alterations in GyrA and GyrB were identified in E. meningoseptica but not in E. anophelis. The QRDR mutation rates were significantly higher in E. meningoseptica than in E. anophelis. These results are compatible with the fact that E. meningoseptica was less susceptible to fluoroquinolones than was E. anophelis.

\section{Conclusions}

Our study reveals that E. anophelis was the predominant human pathogen in the genus Elizabethkingia. Although both species shared similar clinical manifestations, E. meningoseptica and E. anophelis exhibited significantly different antimicrobial susceptibility patterns and possessed diverse mutations in the target gene of fluoroquinolones. Because E. meningoseptica exhibits less susceptibility 
to multiple antibiotics than does E. anophelis, the empirical antimicrobial therapy of E. meningoseptica should be more rigorous and improved to be guided by antimicrobial susceptibility testing.

Supplementary Materials: The following are available online at http:/ / www.mdpi.com/2077-0383/7/12/538/s1, Table S1: Primers and PCR conditions used in this study, Table S2: Information of strains for comparison of $16 \mathrm{~S}$ rRNA and QRDRs in gyrA, gyrB, parC, and parE in this study.

Author Contributions: Conceptualization, J.N.L.; Data curation, J.N.L., C.-H.L., C.-H.Y., and Y.-H.H.; Formal analysis, J.N.L. and Y.-H.H.; Funding acquisition, J.N.L.; Methodology, J.N.L.; Resources, C.-H.L.; Supervision, J.N.L.; Validation, J.N.L., C.-H.L., C.-H.Y. and Y.-H.H.; Writing-Original draft, J.N.L. and C.-H.Y.; Writing-Review \& editing, J.N.L., C.-H.L., C.-H.Y. and Y.-H.H.

Funding: This work was supported by grants EDPJ106075 from E-Da Hospital and MOST 105-2314-B-214-008 and MOST 106-2314-B-214-009-MY2 from the Ministry of Science and Technology, Taiwan.

Conflicts of Interest: The authors declare no conflict of interest.

\section{References}

1. Henriques, I.S.; Araújo, S.; Azevedo, J.S.; Alves, M.S.; Chouchani, C.; Pereira, A.; Correia, A. Prevalence and diversity of carbapenem-resistant bacteria in untreated drinking water in Portugal. Microb. Drug Resist. 2012, 18, 531-537. [CrossRef] [PubMed]

2. Jean, S.S.; Lee, W.S.; Chen, F.L.; Ou, T.Y.; Hsueh, P.R. Elizabethkingia meningoseptica: An important emerging pathogen causing healthcare-associated infections. J. Hosp. Infect. 2014, 86, 244-249. [CrossRef] [PubMed]

3. Nicholson, A.C.; Gulvik, C.A.; Whitney, A.M.; Humrighouse, B.W.; Graziano, J.; Emery, B.; Bell, M.; Loparev, V.; Juieng, P.; Gartin, J.; et al. Revisiting the taxonomy of the genus Elizabethkingia using whole-genome sequencing, optical mapping, and MALDI-TOF, along with proposal of three novel Elizabethkingia species: Elizabethkingia bruuniana sp. nov., Elizabethkingia ursingii sp. nov., and Elizabethkingia occulta sp. nov. Anton. Van Leeuwenhoek 2017, 111, 55-72. [CrossRef] [PubMed]

4. King, E.O. Studies on a group of previously unclassified bacteria associated with meningitis in infants. Am. J. Clin. Pathol. 1959, 31, 241-247. [CrossRef] [PubMed]

5. Teo, J.; Tan, S.Y.-Y.; Tay, M.; Ding, Y.; Kjelleberg, S.; Givskov, M.; Lin, R.T.; Yang, L. First case of E anophelis outbreak in an intensive-care unit. Lancet 2013, 382, 855-856. [CrossRef]

6. Lau, S.K.; Chow, W.N.; Foo, C.H.; Curreem, S.O.; Lo, G.C.; Teng, J.L.; Chen, J.H.; Ng, R.H.; Wu, A.K.; Cheung, I.Y.; et al. Elizabethkingia anophelis bacteremia is associated with clinically significant infections and high mortality. Sci. Rep. 2016, 6, 26045. [CrossRef] [PubMed]

7. Lin, J.N.; Lai, C.H.; Yang, C.H.; Huang, Y.H.; Lin, H.H. Clinical manifestations, molecular characteristics, antimicrobial susceptibility patterns and contributions of target gene mutation to fluoroquinolone resistance in Elizabethkingia anophelis. J. Antimicrob. Chemother. 2018, 73, 2497-2502. [CrossRef]

8. Perrin, A.; Larsonneur, E.; Nicholson, A.C.; Edwards, D.J.; Gundlach, K.M.; Whitney, A.M.; Gulvik, C.A.; Bell, M.E.; Rendueles, O.; Cury, J.; et al. Evolutionary dynamics and genomic features of the Elizabethkingia anophelis 2015 to 2016 Wisconsin outbreak strain. Nat. Commun. 2017, 8, 15483. [CrossRef]

9. Navon, L.; Clegg, W.J.; Morgan, J.; Austin, C.; McQuiston, J.R.; Blaney, D.D.; Walters, M.S.; Moulton-Meissner, H.; Nicholson, A. Notes from the field: Investigation of Elizabethkingia anophelis cluster-Illinois, 2014-2016. MMWR Morb. Mortal Wkly Rep. 2016, 65, 1380-1381. [CrossRef]

10. Lin, J.N.; Lai, C.H.; Yang, C.H.; Huang, Y.H.; Lin, H.F.; Lin, H.H. Comparison of four automated microbiology systems with $16 \mathrm{~S}$ rRNA gene sequencing for identification of Chryseobacterium and Elizabethkingia species. Sci. Rep. 2017, 7, 13824. [CrossRef]

11. Holmes, B.; Steigerwalt, A.G.; Nicholson, A.C. DNA-DNA hybridization study of strains of Chryseobacterium, Elizabethkingia and Empedobacter and of other usually indole-producing non-fermenters of CDC groups IIc, IIe, IIh and IIi, mostly from human clinical sources, and proposals of Chryseobacterium bernardetii sp. nov., Chryseobacterium carnis sp. nov., Chryseobacterium lactis sp. nov., Chryseobacterium nakagawai sp. nov. and Chryseobacterium taklimakanense comb. nov. Int. J. Syst. Evol. Microbiol. 2013, 63, 4639-4662. [CrossRef] [PubMed]

12. Felske, A.; Rheims, H.; Wolterink, A.; Stackebrandt, E.; Akkermans, A.D. Ribosome analysis reveals prominent activity of an uncultured member of the class Actinobacteria in grassland soils. Microbiology 1997, 143, 2983-2989. [CrossRef] [PubMed] 
13. Hantsis-Zacharov, E.; Shakéd, T.; Senderovich, Y.; Halpern, M. Chryseobacterium oranimense sp. nov., a psychrotolerant, proteolytic and lipolytic bacterium isolated from raw cow's milk. Int. J. Syst. Evol. Microbiol. 2008, 58, 2635-2639. [CrossRef] [PubMed]

14. Janda, J.M.; Abbott, S.L. 16S rRNA gene sequencing for bacterial identification in the diagnostic laboratory: Pluses, perils, and pitfalls. J. Clin. Microbiol. 2007, 45, 2761-2764. [CrossRef] [PubMed]

15. Kumar, S.; Stecher, G.; Tamura, K. MEGA7: Molecular evolutionary genetics analysis version 7.0 for bigger datasets. Mol. Biol. Evol. 2016, 33, 1870-1874. [CrossRef]

16. Huson, D.H.; Richter, D.C.; Rausch, C.; Dezulian, T.; Franz, M.; Rupp, R. Dendroscope: An interactive viewer for large phylogenetic trees. BMC Bioinform. 2007, 8, 460. [CrossRef]

17. Clinical and Laboratory Standards Institute. Performance Standards for Antimicrobial Susceptibility Testing, Twenty-Sixth Informational Supplement, 26th ed.; Clinical and Laboratory Standards Institute: Wayne, PA, USA, 2016.

18. Kelesidis, T.; Karageorgopoulos, D.E.; Kelesidis, I.; Falagas, M.E. Tigecycline for the treatment of multidrug-resistant Enterobacteriaceae: A systematic review of the evidence from microbiological and clinical studies. J. Antimicrob. Chemother. 2008, 62, 895-904. [CrossRef]

19. Hung, P.P.; Lin, Y.H.; Lin, C.F.; Liu, M.F.; Shi, Z.Y. Chryseobacterium meningosepticum infection: Antibiotic susceptibility and risk factors for mortality. J. Microbiol. Immunol. Infect. 2008, 41, 137-144.

20. Lin, P.Y.; Chu, C.; Su, L.H.; Huang, C.T.; Chang, W.Y.; Chiu, C.H. Clinical and microbiological analysis of bloodstream infections caused by Chryseobacterium meningosepticum in nonneonatal patients. J. Clin. Microbiol. 2004, 42, 3353-3355. [CrossRef]

21. Lin, Y.T.; Chiu, C.H.; Chan, Y.J.; Lin, M.L.; Yu, K.W.; Wang, F.D.; Liu, C.Y. Clinical and microbiological analysis of Elizabethkingia meningoseptica bacteremia in adult patients in Taiwan. Scand. J. Infect. Dis. 2009, 41, 628-634. [CrossRef]

22. Huang, Y.C.; Lin, Y.T.; Wang, F.D. Comparison of the therapeutic efficacy of fluoroquinolone and non-fluoroquinolone treatment in patients with Elizabethkingia meningoseptica bacteraemia. Int. J. Antimicrob. Agents 2018, 51, 47-51. [CrossRef] [PubMed]

23. Huang, Y.C.; Huang, Y.W.; Lin, Y.T.; Wang, F.D.; Chan, Y.J.; Yang, T.C. Risk factors and outcome of levofloxacin-resistant Elizabethkingia meningoseptica bacteraemia in adult patients in Taiwan. Eur. J. Clin. Microbiol. Infect. Dis. 2017, 36, 1373-1380. [CrossRef] [PubMed]

24. Kämpfer, P.; Matthews, H.; Glaeser, S.P.; Martin, K.; Lodders, N.; Faye, I. Elizabethkingia anophelis sp. nov., isolated from the midgut of the mosquito Anopheles gambiae. Int. J. Syst. Evol. Microbiol. 2011, 61, $2670-2675$. [CrossRef]

25. Frank, T.; Gody, J.C.; Nguyen, L.B.L.; Berthet, N.; Le Fleche-Mateos, A.; Bata, P.; Rafaï, C.; Kazanji, M.; Breurec, S. First case of Elizabethkingia anophelis meningitis in the Central African Republic. Lancet 2013, 381, 1876. [CrossRef]

26. Han, M.S.; Kim, H.; Lee, Y.; Kim, M.; Ku, N.S.; Choi, J.Y.; Yong, D.; Jeong, S.H.; Lee, K.; Chong, Y. Relative prevalence and antimicrobial susceptibility of clinical isolates of Elizabethkingia species based on 16S rRNA gene sequencing. J. Clin. Microbiol. 2017, 55, 274-280. [CrossRef] [PubMed]

27. Jacoby, G.A. Mechanisms of resistance to quinolones. Clin. Infect. Dis. 2005, 41 (Suppl. 2), 120-126. [CrossRef]

(C) 2018 by the authors. Licensee MDPI, Basel, Switzerland. This article is an open access article distributed under the terms and conditions of the Creative Commons Attribution (CC BY) license (http://creativecommons.org/licenses/by/4.0/). 